\title{
Preliminary Assessment Of Potential Water Savings Actions By Cement Cienfuegos SA PML
}

\author{
Juan Jose Cabello Eras ${ }^{a}$, Arturo Alvares Acevedo ${ }^{b}$, Alexis Sagastume Gutiérrez ${ }^{c}$, Jorge Luis Romero Cabrera ${ }^{c}$, Yabiel \\ Pérez Gómez ${ }^{\mathrm{d}}$ \\ ${ }^{a}$ Doctor en Ciencias Técnicas, Universidad de Cienfuegos, Cienfuegos, Cuba, jcabello@ucf.edu.cu \\ ${ }^{b}$ Master en ciencias, Cementos Cienfuegos SA, Cienfuegos, Cuba, aalvarez@ccfg.colombus.cu \\ ${ }^{c}$ Doctor en Ciencias Técnicas, Universidad de Cienfuegos, Cienfuegos, Cuba, asagastume@ucf.edu.cu \\ ${ }^{d}$ Master en Ciencias, Cementos Cienfuegos SA, Cienfuegos, Cuba, d jromero@ccfg.colombus.cu \\ ${ }^{e}$ Master en Ciencias, Universidad de Cienfuegos, Cienfuegos, Cuba, ypgomez@ucf.edu.cu
}

\begin{abstract}
The paper makes a quantitative analysis of the main water consumers processes in the company Cementos Cienfuegos SA, from which actions for savings are proposed through the introduction of reuse, collection and use of rain water. A preliminary assessment of the proposed tasks was made, that led to the conclusion that the company has potential to significantly reduce the cost of its invoices in payment of water therefore it could improve the indicators of specific water consumption.
\end{abstract}

Keywords: cement, water, water reuse, water management.

\section{Evaluación preliminar de las potencialidades de ahorro de agua mediante acciones de PML en "cementos cienfuegos" SA.}

\section{RESUMO}

En el artículo se realiza un análisis cuantitativo de los principales procesos consumidores de agua en la empresa Cementos Cienfuegos SA, a partir del cual se proponen acciones para su ahorro a través de la introducción del reúso; la recolección y el aprovechamiento de las aguas pluviales. Se hizo una evaluación preliminar de las tareas propuestas; que permitieron concluir que la empresa cuenta con potencialidades para reducir de manera significativa los importes de sus facturas por concepto de pago de agua de manera que le permitirían mejorar los indicadores de consumo específico de agua.

Palabras claves: cemento, agua, reutilización del agua, gestión del agua.

\section{INTRODUCCIÓN}

Las plantas cementeras utilizan grandes volúmenes de agua en sus procesos productivos, en la fabricación mediante proceso húmedo se consumen mayores cantidades debido a su utilización para la obtención de la colas y en la fabricación por vía seca el uso está orientado fundamentalmente al enfriamiento de los equipos tecnológicos.

A finales del siglo pasado en las fábricas de cemento se necesitaban entre 0,4 y $0,6 \mathrm{~m}^{3}$ de agua por tonelada de cemento producida, en su mayoría para la refrigeración de las máquinas a través de circuitos cerrados en los que solo se deben reponer las pérdidas y en las plantas que trabajan con método seco para la refrigeración de los gases de escape de los hornos. En las instalaciones que trabajan con el método húmedo se necesitaba aproximadamente otro metro cúbico por tonelada de cemento para la molienda del lodo [2].

En los últimos años la industria cementera ha logrado importantes avances en la eficiencia en la utilización del agua en la fabricación de cemento y varias empresas reportan valores del consumo específico de agua de entre 50 y 360 litros por tonelada [10].

La Fábrica de Cemento de Cienfuegos fue inaugurada en 1982 y en 2001 fue sometida a una reparación capital que dio solución a los principales problemas ambientales que presentaba en su funcionamiento, particularmente las emisiones de polvo y hoy es una empresa reconocida por su buen desempeño ITEGAM - JETIA Vol. 01, No. 01. Março de 2015. Manaus - Amazonas, Brasil. ISSN 2447-0228 (ONLINE). 
ambiental. La tecnología de producción es por vía seca. La Fábrica inicialmente la conformaban tres líneas paralelas de producción con una capacidad instalada de 1500000 ton/año de Clinker, después de la modernización quedo con dos líneas e igual capacidad instalada y su producción ha ido aumentando gradualmente y en 2010 produjo 1016400 ton y el 2011 cerró con 1023479 ton.

Además del consumo de agua en las partes típicas en la fabricación por el método seco en la fábrica se consume agua en las instalaciones no industriales, principalmente el riego de las áreas verdes que constituyen un sistema ambiental destinado a asegurar que el polvo depositado en el terreno no vuelva a suspenderse en el aire, evitar la erosión. También se presenta un consumo social de agua en los baños y en la elaboración de alimentos.

En Cementos Cienfuegos S.A. como parte de su estrategia ambiental se ha establecido un programa de ahorro de agua que en los últimos dos años ha venido disminuyendo sustancialmente los índices de consumo de agua lo que se muestra en la tabla 1 .

Tabla 1. Producción e índice de consumo de agua en 2010 y 2011. Fuente de los datos [1].

\begin{tabular}{ccc} 
Año & 2010 & 2011 \\
\hline Producción (ton) & 1016400 & 1023479 \\
\hline Consumo de agua $\left(\mathrm{m}^{3}\right)$ & 541599 & 358948 \\
\hline $\begin{array}{c}\text { Indice de consumo } \\
\left(\mathrm{m}^{3} / \text { ton }\right)\end{array}$ & 0,53 & 0,35
\end{tabular}

Estos resultados están aún lejos de los mejores reportados por otras fábricas que llegan incluso aconsumir menos de 0,1 m3/ton aplicando criterios de Producción Más Limpia como es reciclado y reutilización del agua.

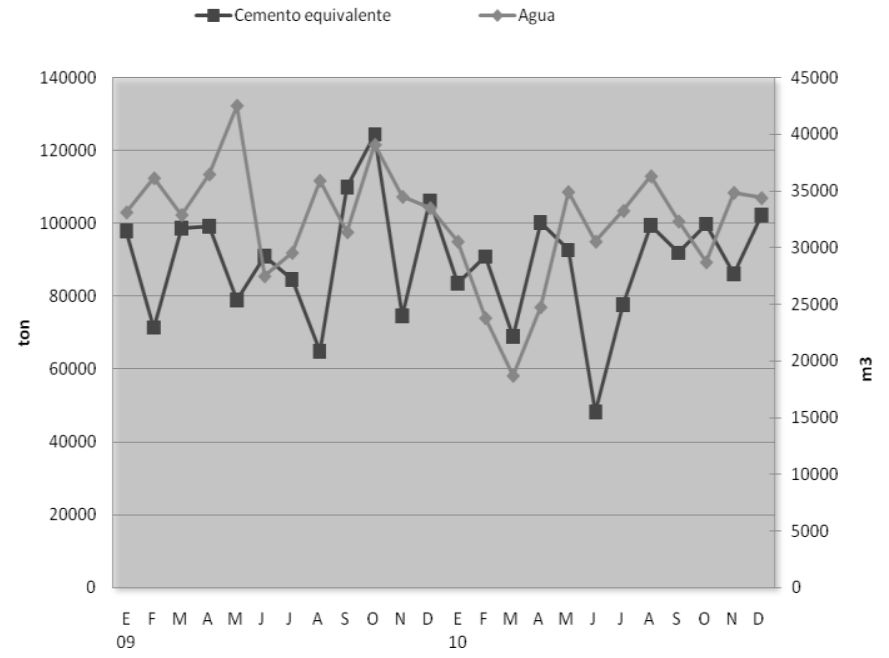

Figura 1. Gráfico de consumo de agua y producción de cemento equivalente datos: [1]
En la Figura 1 se muestra el comportamiento de la producción y el consumo de agua mensual durante 2010 y se aprecia claramente que no hay una correlación entre ambos parámetros, esto evidencia que existen potencialidades de mejora no explotadas en el proceso.

Esta no correlación se encuentra en primer lugar causada por el hecho de que el consumo de agua en la empresa se registra mediante un contador a la entrada a la instalación y por lo tanto incluye el consumo de agua no asociada al proceso, en segundo lugar a malas prácticas e ineficiencias en diferentes etapas de proceso productivo.

El presente trabajo está orientado a la evaluación de las potencialidades de mejora en el uso racional del agua en esta fábrica y a proponer acciones para esto.

\section{DESCRIPCIÓN DEL PROCESO PRODUCTIVO Y BALANCE DEL USO DEL AGUA.}

En la figura 2 se puede apreciar el esquema del proceso productivo de Cementos Cienfuegos a SA, esto comienza con la extracción de la materia prima en las minas y su transporte hacia la estación de trituración y secado, de donde se envía a través de transportadores de banda hacia los silos de almacenamiento.

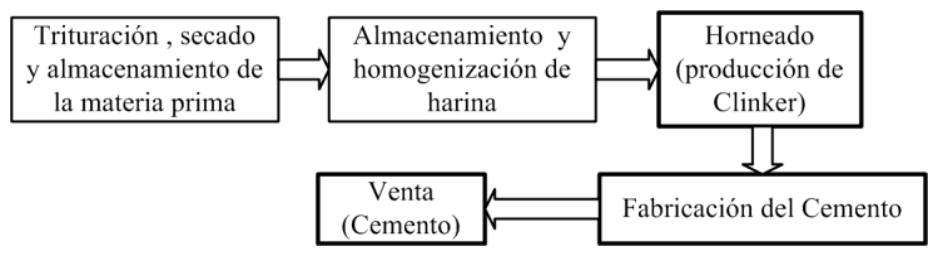

Figura 2. Esquema del proceso productivo de Cementos Cienfuegos SA. Fuente: [1].

El desempolvado en el área de trituración se realiza mediante 2 separadores ciclónicos, a la salida del secador se encuentra instalado el filtro de mangas (casa de bolsas) y en cada torre de transferencia de las bandas transportadoras hasta la fábrica se instalaron filtros de manga.

En el proceso de preparación de materias primas no se utiliza agua. En el área se encuentra enclavado un comedor para los doce trabajadores que operan la instalación, que cuenta además con dos instalaciones sanitarias, dos bebederos, que entre todos consumen un total promedio de 9000 litros de agua semanales.

Las materias primas adecuadamente dosificadas son conducidas a la estación de molienda de crudo, donde pasan por un triturador secador de impacto, el grueso pasa al molino de bolas, el polvo de arrastre pasa al separador, y el fino pasa a la batería de ciclones. El material separado pasa a un sinfín recuperador y el aire pasa a la torre estabilizadora donde se enfría y se envía al electrofiltro. El material separado es enviado a los 
silos de almacenaje para su homogenización. En esta etapa se consume agua en el enfriamiento de las chumaceras y los reductores de los molinos, la instalación cuenta con tres molinos y el consumo de cada uno es de $30 \mathrm{~m} 3$ de agua por hora de trabajo, por lo que el consumo total de la instalación es de $90 \mathrm{~m} 3$ por hora.

De los silos de almacenaje, la harina homogeneizada es conducida al precalentador, y de ahí pasa al horno rotatorio donde ocurre la se transformación en Clinker. Posteriormente a través de un transportador de cangilones el Clinker es enviado a los silos de almacenamiento.

En el proceso de pirolización de la harina se consume agua en el enfriamiento de las chumaceras y rolos del horno a través de un circuito cerrado que tiene incorporada una torre de enfriamiento.

El flujo de gases calientes con alto contenido de polvo que sale del horno es filtrados a través de un sistema compuesto por tres electrofiltros, antes de la entrada a estos el flujo pasa a través de tres torres estabilizadoras donde es rociado con agua para reducir su temperatura y mejorar sus propiedades eléctricas, lo que aumenta la efectividad del filtrado.

Para el rociado en las torres estabilizadoras se utiliza agua proveniente del circuito cerrado de refrigeración de la maquinaria, siendo esta la mayor fuente de pérdidas a reponer en este ya que el agua rociada en su mayor parte se evapora y transfiere a la atmósfera con los gases de escape.

Tabla 2. Balance de agua de las instalaciones.

\begin{tabular}{|c|c|c|}
\hline $\begin{array}{l}\text { Sistema } \\
\text { Tecnológico }\end{array}$ & \multicolumn{2}{|c|}{ Consumo, $\left[\mathrm{m}^{3} / \mathrm{h}\right]$} \\
\hline & Entrada & Salida \\
\hline \multicolumn{3}{|c|}{ Molinos de crudo } \\
\hline 361-MB1 & 30 & 30 \\
\hline 362-MB1 & 30 & 30 \\
\hline 363-MB3 & 30 & 30 \\
\hline \multicolumn{3}{|c|}{ Hornos } \\
\hline Horno Línea I & 33 & 33 \\
\hline Horno Línea II & 21.3 & 21.3 \\
\hline \multicolumn{3}{|c|}{ Molino de Petcoke } \\
\hline $\begin{array}{c}\text { Enfriamiento del } \\
\text { sistema de lubricación }\end{array}$ & 50.8 & 50.8 \\
\hline \multicolumn{3}{|c|}{ Torres acondicionadoras } \\
\hline 421-TA1 & 8 & \\
\hline 422-TA1 & 12 & \\
\hline 423-TA1 & 10 & \\
\hline \multicolumn{3}{|c|}{ Sistema de aire comprimido. } \\
\hline Sala de compresores & 341.5 & 341.5 \\
\hline \multicolumn{3}{|c|}{ Perdidas en la torre de enfriamiento $(3 \%)$} \\
\hline Reposición & 19,8 & \\
\hline
\end{tabular}

Fuente de los datos: [1].
El circuito cerrado de refrigeración de la maquinaria también tiene incorporado a la estación de compresores y el molino de petcoke utilizado para la preparación de este combustible utilizado en el horno.

El agua no asociada directamente al proceso incluye además el riego a los jardines, el remojado de las pilas de petcoke para evitar la emisión de polvo y mantener su temperatura por debajo de 70 grados centígrados.

En la tabla 2 se resumen el balance del consumo de agua en estas instalaciones, el total de la demanda de agua de enfriamiento en el circuito cerrado es de $566,6 \mathrm{~m} 3 / \mathrm{h}$, las perdidas producto del rociado en las torres de estabilización es del orden los $30 \mathrm{~m} 3 / \mathrm{h}$ y las perdidas en las torre de enfriamiento según sus parámetros de diseño son de 19,8 m3/h. La significación anual de estas pérdidas se muestra en la tabla 3 .

Tabla 3. Resumen del consumo de agua en las torres estabilizadoras y de enfriamiento.

\begin{tabular}{lccc} 
& \multicolumn{2}{c}{ Línea II } & Línea \\
\cline { 2 - 4 } & $\begin{array}{c}\text { Torre III } \\
\text { III }\end{array}$ & $\begin{array}{c}\text { Torre II } \\
\text { II }\end{array}$ & $\begin{array}{c}\text { Torre I } \\
\text { I }\end{array}$ \\
\hline Consumo de agua $\mathrm{m}^{3} / \mathrm{h}$ & 10 & 12 & 8 \\
\hline Tiempo de operación $\mathrm{h} / \mathrm{d}$ & 12 & 12 & 12 \\
\hline${\text { Consumo anual } \mathrm{m}^{3}}^{3}$ & 39600 & 47520 & 31680
\end{tabular}

Consumo anual en las torres 118800

\begin{tabular}{lc}
\hline & Torre de enfriamiento \\
\hline Pérdidas de agua $\mathrm{m}^{3} / \mathrm{h}$ & 19,8 \\
\hline Tiempo de operación $\mathrm{h} / \mathrm{d}$ & 12 \\
\hline Consumo anual torre de & 76032 \\
enfriamiento, $\mathrm{m}^{3}$ & \\
\hline Fuente: elaboración propia. &
\end{tabular}

El consumo de agua no industrial tiene tres componentes principales:

- Rociado le las pilas de carbón el que se dedican $3 \mathrm{~m} 3$ por hora diurna con el objetivo de mantener su temperatura por debajo de los 70 grados centígrados y reducir la emisión de polvo.

- Riego de las áreas verdes, a lo que se dedica según los estimados agronómicos de 15000 m3/año y se realiza con agua proveniente del circuito de enfriamiento de las maquinas.

- Consumo social en los baños e instalaciones sanitarias, oficinas, laboratorios y elaboración de los alimentos. Este consumo no se mide pero se puede estimar a partir del balance de agua, en el año 2010 restando a los 358943 m3 de agua registrados a la entrada se restan los $194000 \mathrm{~m} 3$ de pérdidas en el circuito de enfriamiento, los $15000 \mathrm{~m} 3$ dedicados al riego y los $9000 \mathrm{~m} 3$ dedicados rociar las pilas de carbón y se obtiene un estimado de $150000 \mathrm{~m}^{3}$. 
Según estos cálculos el índice de consumo considerando solo el agua utilizada directamente en el proceso de fabricación de cemento estaría en el orden de los 0,19 $\mathrm{m} 3 /$ ton, más cercano a los resultados internacionales.

\section{EVALUACIÓN DE LA REUTILIZACIÓN DE AGUA EN EL PROCESO.}

La evaluación de la reutilización de agua en el proceso estará orientada a independizar el suministro del agua para las torres estabilizadoras de los electrofiltros y para el riego del circuito cerrado de refrigeración de la maquinaria de donde proviene actualmente. Lo que permitirá buscar fuentes alternativas para este consumo.

En las torres estabilizadoras el agua es rociada directamente al flujo de polvo y gases antes del electrofiltro, desde un tanque de $1000 \mathrm{~m} 3$ (independiente para cada torre, el flujo de agua es regulado por una válvula automática en función del gasto másico de gases y su temperatura a la entrada de la torre). Esta agua proviene del circuito cerrado de agua de enfriamiento a través de bombas situadas en la torre de enfriamiento, las que se encargan de mantener el nivel del tanque mediante un sistema automático de control del nivel y recirculación nuevamente a la torre de enfriamiento.

Tabla 4. Valores comparativos de la composición del agua de enfriamiento y salida de la laguna de oxidación.

\begin{tabular}{ccccc} 
Parámetros & $\mathrm{U} / \mathrm{M}$ & $\begin{array}{c}\text { Salida de la } \\
\text { laguna }\end{array}$ & $\begin{array}{c}\text { Agua } \\
\text { tecnológi }\end{array}$ \\
\hline Fósforo Total & $\mathrm{mg} / \mathrm{L}$ & 0.276 & \pm 0.062 & 0.765 \\
\hline NTK & $\mathrm{mg} / \mathrm{L}$ & 0.97 & \pm 0.38 & - \\
\hline DQO & $\mathrm{mg} / \mathrm{L}$ & 56 & \pm 10.1 & - \\
\hline DBO5 & $\mathrm{mg} / \mathrm{L}$ & 27.5 & \pm 8.8 & - \\
\hline $\begin{array}{c}\text { Sólidos } \\
\text { sedimentables }\end{array}$ & $\mathrm{ml} / \mathrm{L}$ & $<0.2$ & --- & $<0.01$ \\
\hline $\begin{array}{c}\text { Sólidos } \\
\text { Suspendidos }\end{array}$ & $\mathrm{mg} / \mathrm{L}$ & 38.8 & \pm 7.8 & $<1$ \\
\hline OD & $\mathrm{mg} / \mathrm{L}$ & 8.12 & \pm 0.12 & - \\
\hline pH & $\mathrm{Unidades}$ & 7.43 & \pm 0.07 & $7.5-8.5$ \\
\hline de $\mathrm{pH}$ & & & \\
\hline Temperatura & ${ }^{0} \mathrm{C}$ & 25.3 & \pm 0.2 & \\
\hline Ce & $\mu \mathrm{s} / \mathrm{cm}$ & 502 & \pm 12.6 & 1000 \\
\hline Grasas y Aceites & $\mathrm{mg} / \mathrm{L}$ & 249 & \pm 81 & $<100$ \\
\hline $\begin{array}{c}\text { Dureza Total } \\
\mathrm{mg} / \mathrm{L}\end{array}$ & --- & --- & $0.2-0.4$
\end{tabular}

Fuente: Registro ambiental de Cementos Cienfuegos S.

La propuesta concreta consiste en independizar el sistema de suministro de agua para las torres estabilizadoras, del circuito de agua de enfriamiento directamente en el colector de salida de la torre de enfriamiento y suministrar a las torres y eventualmente para el riego el agua de salida del sistema de lagunas de oxidación de la planta, al que se encuentran conectado además otras instalaciones cercanas.

La cuantificación de la emisión del sistema de lagunas realizadas por la Autoridad Ambiental es del orden de los 260 $000 \mathrm{~m} 3 / a n ̃ o$, siendo obligatorio según las regulaciones vigente verter al medio el $60 \%$, lo que deja disponibles 100000 m3/año de agua.

En la tabla 4 se puede apreciar la comparación entre los parámetros de agua utilizada en el circuito cerrado de enfriamiento y el agua de salida del sistema de lagunas de oxidación.

De la tabla anterior se concluye que las características del agua tecnológica difieren respecto al agua de salida de la laguna en los sólidos sedimentables y las grasas y aceites respectivamente, siendo superiores a los exigidos para el sistema de agua de enfriamiento del circuito cerrado de la planta, desde donde se alimenta el sistema de rociado de las torres estabilizadoras de los electrofiltros por lo que se hace necesario su tratamiento mediante una estación de tratamiento adicional. En la figura 3 se muestra el esquema del circuito de suministro que se requerirá para implementar esta solución.

En el diagrama de la figura 3 se aprecia que para el bombeo de agua desde la instalación de tratamiento hacia los tanques de consumo de las torres estabilizadoras serán colocadas tres bombas (una trabajando, una en reserva activa y otra en mantenimiento) con el objetivo de garantizar la seguridad del trabajo de las torres.

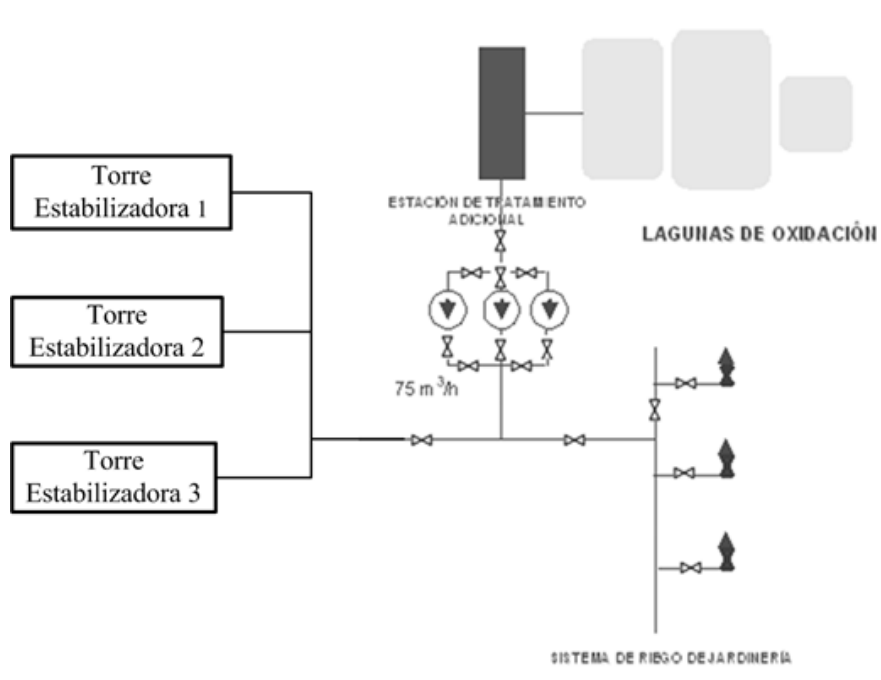

Figura 3. Circuito para el suministro de agua reutilizada a las torres estabilizadoras.

Por otro lado se interconectará a esta línea una derivación para el suministro de agua a los rociadores de la jardinería, el objetivo es mantener un flujo de agua continuo a la 
planta de tratamiento, ya que el consumo de agua de las torres no tiene un comportamiento estable en el tiempo, por lo que existen excedentes de agua en determinados momentos que de no utilizar en la jardinería tendría que ser descargado a la cuenca.

En general la reutilización del agua residual a la salida de las lagunas de oxidación tendría un significativo aporte económico ya que permite recuperar un volumen de agua estimado del orden de los $100000 \mathrm{~m} 3 /$ año a un costo de 0.30 $\$ / \mathrm{m} 3$, representa $30000 \$ /$ año de ahorro.

Tabla 4. Evaluación preliminar de factibilidad de la inversión para la reutilización del agua.

Costos de inversión y mantenimiento.

\begin{tabular}{lc}
\hline Bombas unidad $\quad(1000 \$ \times 3)$ & $3000.00 \$$ \\
\hline Tuberías $(1000 \mathrm{~m} \times 1.56 \$)$ & 1560.00 \\
\hline Sistema de tratamiento & $26000.00 \$$ \\
\hline Costo total del equipamiento & $\mathbf{3 0 5 6 0 . 0 0 \$}$ \\
\hline Mano de obra & $1200.00 \$$ \\
\hline Insumos & $1500.00 \$$ \\
\hline Costo total de instalación & $\mathbf{2 7 0 0 . 0 0 \$}$ \\
\hline Inversión total & $\mathbf{3 3 2 6 9} \$$ \\
\hline Costos de operación anuales & $\mathbf{4 2 0 0} \$$ \\
\hline
\end{tabular}

Fuente: elaboración propia.

De este cálculo preliminar se concluye que la inversión se pagaría en poco más de un año, la inyección de alrededor de $100000 \mathrm{~m} 3$ de agua reutilizada permitiría un estimado de reducción del índice de consumo general de agua en la planta contra agua facturada a $0,25 \mathrm{~m} 3 /$ ton y del índice de consumo productivo respecto al agua facturada para el año 2111 a 0,19 $\mathrm{m} 3 /$ ton.

\section{EVALUACIÓN DE LA POTENCIALIDAD DE APROVECHAMIENTO DEL AGUA PLUVIAL.}

La captación de agua de lluvia es un medio relativamente simple de obtener agua para consumo de planta. En muchas industrias del mundo con alta o media precipitación $\mathrm{y}$ en donde no se dispone de agua en cantidad y calidad necesaria para las operaciones tecnológicas, se recurre al agua de lluvia como fuente de abastecimiento. Al efecto, el agua de lluvia es interceptada, colectada y almacenada en depósitos para su posterior uso. En la captación del agua de lluvia se acostumbra a utilizar la superficie de las edificaciones industriales como captación, conociéndose a este modelo como SCAPT (sistema de captación de agua pluvial en techos). Este modelo tiene un beneficio adicional es que además de su ubicación minimiza la contaminación del agua.

La captación de agua de lluvia presenta como ventajas que no requiere energía para la operación de recolección, facilidad de mantenimiento, comodidad y ahorro de tiempo en la recolección del agua de lluvia.
En el proyecto inicial del emplazamiento de la planta se establece que la totalidad del área productiva se encuentra ubicada sobre una red de recolección de aguas pluviales mediante canales y sistemas de captación desde las edificaciones con el objetivo de excluir las posibles inundaciones internas y canalizar estas aguas hacia el canal de incorporación a la cuenca.

Este canal de salida está conectado a una depresión del terreno con profundidad media de $17 \mathrm{~m}$ y un área de $90 \mathrm{~m}$ x 85 $\mathrm{m}$, con un volumen de llenado máximo estimado de 130000 m3según los datos del proyecto civil.

Para la determinación del volumen de agua anual recolectada se parte de la determinación de la precipitación promedio mensual a partir de los datos promedio mensuales de precipitación de los últimos 10 o 15 años. Este valor se expresa en los litros por metro cuadrado y por mes que es capaz de colectarse en la superficie horizontal del techo.

En el caso de Cementos Cienfuegos S.A. las precipitaciones tienen dos períodos bien definidos: lluvioso de mayo a octubre y seco de noviembre a abril, con una media anual de 1400 a 1500 $\mathrm{mm}$. El promedio para el período lluvioso es de 900 a $1000 \mathrm{~mm}$ y para el período seco es de 200 a $250 \mathrm{~mm}$. El promedio de días con lluvia en el año es de 132 días.

Considerando una relación de recolección establecida para edificaciones y plazoletas donde $25 \mathrm{~mm}$ de lluvia en $1 \mathrm{~m} 2$ recolecta 8.55 litros de agua lluvia, las instalaciones de Cementos Cienfuegos S.A. con un área no verde de $330000 \mathrm{~m} 2$ y una media anual de $1450 \mathrm{~mm}$ de precipitación anual, tiene potencialidad de acumular $163647 \mathrm{~m} 3$ de agua, de estos seria obligatorio según las regulaciones vigentes aportar el $60 \%$ a la cuenca lo que permitiría disponer en una año de comportamiento normal de las lluvias de un estimado de 90000 m3 para su utilización como agua industrial .

El embalse de esta agua permite ahorrar por concepto de reducción de la factura de agua de 27000 \$. Para implementar este sistema solo se necesita restablecer la cortina de la depresión donde se almacenaría el agua aproximadamente $5 \mathrm{~m}$ de longitud y un espesor de $4 \mathrm{~m}$ y altura de $3.7 \mathrm{~m}$, para lo que es necesario un volumen de $150 \mathrm{~m} 3$ de áridos, que pude ser extraído de las propias canteras de la fábrica y con la maquinaria propia a un costo mínimo para la empresa.

\section{CONCLUSIONES}

La fábrica Cementos Cienfuegos SA tiene potencialidades para reducir a la mínima expresión la facturación de agua para la fabricación de cemento y mejorar notablemente sus indicadores de consumo. 
El uso de agua con fines no productivos debe ser objeto de atención al tener una elevada significación en el consumo general de la fábrica, del orden del $40 \%$.

\section{REFERENCIAS BIBLIOGRÁFICAS.}

[1] Álvarez Arturo. Propuesta de acciones de producción más limpia para el ahorro de agua en Cementos Cienfuegos SA. Tesis de maestría. Universidad de Cienfuegos. 2011.

[2] Ministerio Federal de Cooperación Económica y Desarrollo (BMZ). 1995. Guía de protección ambiental. Material auxiliar para la identificación y evaluación de impactos ambientales. Ed Agencia Alemana de Cooperación al Desarrollo (BTZ) .Eschbom. Alemania.

[3]

http://ces.iisc.ernet.in/energy/HC270799/HDL/ENV/envsp/V ol232.htm

[4] Holcim Colombia S.A. Ecoprocesamiento. http://www.ecoprocesamiento.com/noticia5.html último acceso Junio2014.

[5] Red Interinstitucional de Tecnologías Limpias (RITL). Colombia. Reconversión tecnológica en la planta nobsa cementos boyaca s.a.

www.tecnologiaslimpias.org/html/archivos/casos/Caso\%20ID22 . doc último acceso Junio2014.

[6] Titan SA. Corporate Social Responsibility and Sustainability Report 2010. http://report2010.titan.gr/en/environmental-footprint/waterconsumption/ último acceso Enero 2014.

[7] Energy Technology Systems Analysis Programme (ETSP). Cement Production. http://www.iea- etsap.org/web/ETechDS/PDF/I03_cement_June\%202010_GS-gct.pdf último acceso Junio2014.

[8] Cement Australia. Environmental performance.Water management.http://www.cementaustralia.com.au/wps/wcm/c onnect/website/cement/home/sustainabl edevelopment/environmental-performance/último acceso Junio2014.

[9] Irish cement. Water consumption .http://www.irishcement.ie/sustainabledevelopment/sustainability-performance/waterconsumption/últimoaccesoJunio2014.

[10] Binani Cement Limited. Environmental statement for the financial year ending the 31st March 2012.http://www.binaniindustries.com/csr/pdf/envstatement2011-2012-new-cement-plant.pdfúltimo acceso Junio 2014. 